\title{
El empleo de la tecnología BIM en la docencia vinculada a la Arquitectura. Aprendizaje cooperativo y colaborativo basado en Proyectos reales entre diferentes asignaturas The use of BIM technology in teaching related to Architecture. Cooperative and collaborative learning based on real Projects between different subjects
}

\author{
Leon, I. ; Sagarna, M. ${ }^{a}$; Mora, F. ${ }^{a}$; Marieta, C. ${ }^{\text {b; Otaduy, J. }}{ }^{a}$

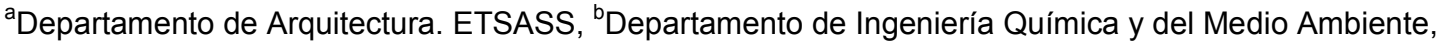 \\ Universidad del País Vasco (UPV-EHU), España, inigo.leon@ehu.eus
}

\begin{abstract}
In this article, it is presented the experience of the Educational Innovation Project accepted by the Basque Country University, which is being developed since 2014 at the Polytechnic University School in Donostia. This project highlights for being the first teaching experience in the Technical Architecture Degree, where teams of teachers from different subjects are developing a work in a cooperative, joint, coordinated and collaborative way, and encompassing the full spectrum of the design - construction process closely with the architecture professional dynamics. BIM technology (Building Information Modeling) is being used so that the same three-dimensional parametric modeling is shared among different subjects, for the resolution of real Learning Based Projects, linking teaching and labor market
\end{abstract}

Keywords: BIM, Architecture, Teaching innovation, active methodologies.

\begin{abstract}
Resumen
En esta comunicación, se presenta la experiencia del Proyecto de Innovación Educativa aceptado por la Universidad del País Vasco, que se está desarrollando desde 2014 en la Escuela Universitaria Politécnica de Donostia. Destaca por ser la primera experiencia docente en el Grado en Arquitectura Técnica, donde equipos docentes de diversas materias están desarrollando un trabajo de manera cooperativa, conjunta, coordinada y colaborativa, abarcando el espectro completo del proceso proyectual-constructivo en estrecha relación con la dinámica profesional Arquitectónica. Se está empleando la tecnología BIM, (Building Information Modeling) de manera que se comparte un mismo modelado tridimensional paramétrico entre diferentes asignaturas, para la resolución del Aprendizaje Basado en Proyectos reales, enlazando docencia y mercado laboral.
\end{abstract}

Palabras clave: BIM, Arquitectura, Innovación docente, metodologías activas. 


\section{Introducción}

En la década de los 90 se produjo una revolución en el sector de la Arquitectura y Construcción, por el cambio en la forma de generar la documentación de un proyecto, pasando de las herramientas manuales a las herramientas digitales empleando aplicaciones CAD (Penttilä y Weck, 2006). Sin embargo, la metodología de trabajo no varió mucho ya que se siguió dibujando de forma geométrica y no paramétrica. El principal problema de esa forma de trabajar, es que se generan muchas representaciones de un mismo modelo, completamente inconexas e independientes entre sí. Este hecho, por desgracia, es bastante común en los proyectos de Arquitectura que, consecuentemente, repercuten de forma negativa durante la ejecución de la obra.

BIM (Building Information Modeling), es una nueva tecnología para la construcción. Se trata de un proceso de generación y gestión de datos del edificio durante su ciclo de vida, utilizando software dinámico de modelado de edificios en 3D y en tiempo real, disminuyendo la pérdida de tiempo y recursos en el diseño y la construcción. La evolución de CAD a BIM se da, no sólo como una herramienta de diseño, sino como un método de trabajo en el cual toda la información se aglutina en un modelo tridimensional. De esta manera se enlazan, por primera vez, tanto la fase conceptual (confección del proyecto) como la de ejecución en obra (Eastman et al. 2011).

Este proceso produce el modelado de información del edificio: No sólo es un simple modelo 3D en un ordenador, es un EDIFICIO VIRTUAL que contiene además con gran detalle información adicional sobre los materiales del edificio y sus características. Es una base de datos tridimensional que hace un seguimiento de todos los elementos que componen el edificio. Esta información puede incluir área y volumen de superficies, propiedades térmicas, descripciones de las habitaciones, precios, información sobre especificaciones de producto. Abarca la geometría del edificio, las relaciones espaciales, la información geográfica, así como las cantidades y las propiedades de los componentes del edificio lo que permite ir cubicando y calculando costos al mismo tiempo que se va proyectando.

El modelo está compuesto de elementos con propiedades físicas: dado que en lugar de usar líneas, círculos, etc. se representan elementos físicos tales como muros, techos, puertas, ventanas, etc. éstos tienen la particularidad de que pueden dotarse de propiedades físicas tales como materiales, acabados, precios, entre otros y luego establecer operaciones e informes con ellos.

El 14 de julio de 2015, el Ministerio de Fomento constituyó la Comisión para la implantación de la metodología BIM en el sector de la construcción española. Esta comisión, se creó teniendo en cuenta que en la Unión Europea, el Parlamento ya ha instado a los países miembros para que aborden la modernización de las normativas de contratación y licitaciones públicas, considerando la conveniencia de incorporar la tecnología BIM para modernizar y mejorar los procesos. Como objetivos de dicha comisión, se hallan el establecer un calendario para adaptación de la normativa para su empleo generalizado, desarrollar los estándares nacionales que posibiliten su uso homogéneo y realizar el mapa académico de formación de esta metodología en España.

Por tanto, el empleo de la tecnología BIM en la enseñanza, al comienzo del proyecto de innovación educativa que se está desarrollando era una propuesta novedosa, pero tras la creación de la Comisión de Fomento, es un paso imprescindible e irrenunciable en la docencia vinculada a la Arquitectura. 
Resulta imperativo el desarrollo y aplicación de modelos de producción que promuevan el trabajo multidisciplinario y colaborativo entre todos los actores involucrados en el proceso de construcción. Es por ello que el camino a recorrer en la docencia ha de ir de la mano, a través de un Aprendizaje Cooperativo y Colaborativo basado en Proyectos reales entre diferentes asignaturas.

\section{El proyecto de innovación docente}

El Proyecto de Innovación Educativa propuesto surgió a la luz de la revolución tecnológica que ha transformado la sociedad actual (Prensky, 2001), en el que resulta imperativo el desarrollo y aplicación de modelos de producción que promuevan el trabajo multidisciplinario y colaborativo entre todos los actores involucrados en el proceso de construcción.

El proyecto que se está desarrollando se basa en el modelo IKD, un modelo cooperativo propio de la UPV-EHU, que pone su acento en que los estudiantes sean los dueños de su aprendizaje y sean formados de forma integral, flexible y adaptada a las necesidades de la sociedad. Se atiende a la necesidad social de un cambio de hábitos con criterios de sostenibilidad económica y social, que haga redirigir la dinámica docente y profesional en el ámbito de la Arquitectura y de la construcción.

El proyecto establece el centro de gravedad en el aprendizaje del alumnado. A través de metodologías activas y con el apoyo de las tecnologías de la información y comunicación.

\subsection{Marco de referencia, IKD.}

Se está incidiendo positivamente sobre los siguientes indicadores del modelo educativo IKD:

\subsubsection{Area1: desarrollo curricular}

Tasa de abandono: Se ha podido constatar que algunas asignaturas del Grado en Arquitectura Técnica presentan una tasa de abandono superior a la definida en los compromisos del centro. Como solución, se están desarrollando proyectos por medio de Edificios Virtuales compartidos entre todas las asignaturas del PIE, de manera que el alumnado pasa de ver el aprendizaje de las materias como una suma de hitos, a un todo, en el que no tiene sentido dejar de matricularse en algunas asignaturas, ya que todas están enlazadas en un mismo conjunto proyectual.

\subsubsection{Area3: desarrollo territorial y social}

Difusión del conocimiento: Hay que tener en cuenta que el profundo descenso en la actividad constructiva en el Estado y su reflejo en nuestra Comunidad Autónoma, ha producido una reducción del alumnado con intención de desarrollo de grados vinculados con la ConstrucciónArquitectura. El PIE tiene un objetivo claro, de difusión de resultados y de las mejoras en tiempos, costos y sobre todo en precisión del proyecto constructivo edificatorio.

\subsection{Objetivos}

Concretando, entre los objetivos principales, está la mejora de la docencia de los grados que 
se imparten en la Escuela Politécnica de Donostia y, esto se concreta de manera resumida en una serie de aspectos: Participacion activa del estudiante en su aprendizaje, utilización de un sistema integrado de información, integrar diferentes materias del grado en un mismo ejercicio común, intensificar el desarrollo de las competencias tranversales del Grado, generar una documentación de proyecto más precisa y adaptarse al nuevo paradigma exigido en la UE por medio de la tecnología BIM.

Esta innovación utiliza metodologías activas basadas en Proyectos, para facilitar la adquisición de las competencias de Grado. Se está coordinando la participación activa del estudiante en la generación del modelo tridimensional único compartido, y en la configuración de toda la documentación vinculada a las diferentes materias que se integran, y que forman parte de un mismo proyecto de edificación.

Se está logrando una mejora en el modelo de enseñanza-aprendizaje de cada una de las asignaturas, ya que se pasa a trabajar con un sistema integrado de información donde cualquier cambio que se ha hecho en cualquier lugar en cualquier momento es automáticamente coordinado a través MODELO VIRTUAL del proyecto entero.

Gracias a la coordinación de los diferentes equipos docentes de tercer curso, esta nueva metodología de trabajo está permitiendo la integración de diferentes materias del grado, en un único modelo tridimensional. Este modelado, está permitiendo que ya desde el 3er curso desarrollen el proyecto edificatorio-constructivo, como un todo interrelacionado, incidiendo especialmente en un mejor desarrollo de los Trabajos de Fin de Grado.

Con este nuevo sistema de trabajo, además de asegurar la adquisición de las competencias específicas de las asignaturas que intervienen en el proceso, se pretende incidir principalmente en el desarrollo de dos de las competencias transversales de la titulación. Por un lado la T2, de trabajo en equipo, y por otro, la T3 (MEC4), para transmitir información oral, gráfica y escrita, incidiendo en un lenguaje constructivo cada vez más preciso, y con especial atención a la transmisión de la información gráfica, base del modelo tridimensional, y de la producción de los planos del proyecto.

Deliberadamente se quiere fomentar la aplicación de la metodología BIM en la línea curricular de la Arquitectura, al permitir a los estudiantes experimentar los beneficios del uso de BIM en un contexto de colaboración, más que como una mera herramienta de modelado y documentación, como sucedió antes. Los estudiantes aprenderán a trabajar juntos, para intercambiar información a través de los modelos BIM y aplicar BIM como parte del proceso de diseño (Boeykens y otros 2013). De esta manera, el alumnado podrá ir alcanzando competencias que se adecúen a esta nueva realidad de mercado. Las tecnologías facilitadoras BIM deben integrarse en los planes de estudios universitarios, no sólo como otro conjunto de herramientas de modelado de diseño y gestión, sino como una manera de investigar y reflexionar sobre la naturaleza cambiante de la profesión de la construcción con el fin de preparar a los estudiantes para estos cambios (Gu y De Vries 2012).

\subsection{Metodología}

El proyecto se está desarrollando entre 4 asignaturas de $3^{\circ}$ del Grado. Las asignaturas en las que se coopera utilizando y compartiendo un mismo modelado 3D BIM de información son: Proyectos Técnicos, Expresión Gráfica Arquitectónica III, Mediciones y Presupuestos, siendo la asignatura de Construcción III la que está sirviendo de potenciador del nexo de unión. 
El equipo de trabajo está compuesto básicamente por docentes con amplia experiencia en proyectos Arquitectónicos, donde cualquiera de los Arquitectos que componemos el grupo, puede resolver las dudas que el alumnado tenga en su modelo tridimensional, en la materia que considere. Por tanto, la forma de tutorización del alumnado adquiere una nueva dimensión, de manera que las tutorías ya no son una sucesión de consultas inconexas con diferentes docentes. Uno de los objetivos de este grupo es que las tutorías realizadas sobre la base del modelado 3D, puedan ser atendidas con un carácter global y unitario, reduciendo los tiempos de ejecución de los proyectos, de manera que el alumnado comprenda el proceso constructivo en su conjunto, en cada paso que dé, y este pueda ser evaluado de forma continua y de manera integradora e interdependiente.

El Proyecto de Innovación Educativa, esta generando en un principio, conjuntos de Edificios Virtuales, que contendrán información adicional sobre los materiales del edificio y sus características. Será una base de datos tridimensional que hace un seguimiento de todos los elementos que componen el edificio. El software utilizado está siendo el REVIT de Autodesk. EI profesorado implicado en este proyecto ha tenido que formarse específicamente en estas tecnologías para poder ofrecer al alumno una herramienta que permita ver la interacción de las competencias entre distintas asignaturas y para formar al alumnado en la utilización de una herramienta imprescindible en un futuro.

Para poder desarrollar la tareas, se crean entre el alumnado desde el comienzo del curso, equipos de trabajo para la generación de los modelados tridimensionales, que servirán para el desarrollo de las diferentes materias incluidas en el proyecto. El trabajo de los equipos incluirá los diversos ingredientes que se consideran básicos para generar actitudes positivas, compromiso y crecer en valores: interdependencia positiva, interacción positiva cara a cara, exigibilidad individual, responsabilidad personal, habilidades cooperativas y autoanálisis de grupo entre otras.

Como resultado de aprendizaje, en la asignatura de Proyectos Técnicos el alumnado habrá diseñado un edificio de viviendas de protección oficial (con el cumplimiento de la normativa vigente como CTE, EHE08, accesibilidad, etc.) en una parcela dada, utilizando la herramienta REVIT. Utilizando la misma herramienta y partiendo del ejercicio ya descrito el alumnado dibuja e imprime los planos que deberán cumplir lo establecido en la asignatura Expresión Gráfica III. Además realiza las mediciones de las diferentes partidas, para poder presupuestar el proyecto, obteniendo así el documento final a entregar en la asignatura Mediciones y Presupuestos. La asignatura de Construcción III sirve de potenciador del nexo de unión.

Para poder gestionar el modelo tridimensional compartido que sirve de base para el desarrollo del proyecto, es de gran importancia el uso de una plataforma digital donde los diferentes integrantes del equipo puedan acceder al modelo común. Inicialmente se planteó el uso de Moodle o "e-gela", pero este sistema presenta varios inconvenientes para la gestión del modelo, ya que por un lado los archivos superan con facilidad los $10 \mathrm{Mb}$, y por otro, estas plataformas no permiten que el modelo sea sincronizado, por ello, se han utilizado sistemas alternativos, y aún se están probando nuevos sistemas que están permitiendo la sincronización en tiempo real.

\subsection{Evaluación de Resultados}

Para evaluar el éxito del proyecto de innovación educativa se están empleando 2 vertientes. Por un lado, se han establecido una serie de rubricas de evaluación entre las 4 asignaturas, 
que sirven para testar la mejora en el aprendizaje del alumnado. Si tenemos en cuenta las entregas iniciales desarrolladas, podemos afirmar que la tasa de aprobados en las asignaturas se ha elevado, incluso en un $15 \%$, dándose casos como en la asignatura de Expresión Gráfica III, donde se ha alcanzado un $100 \%$ de aprobados.

Por otro, se han establecido una serie de encuestas de satisfacción tanto entre el alumnado como del profesorado del Grado. En general, los resultados de las encuestas pasadas a comienzos de Diciembre de 2015, demuestran una clara satisfacción del alumnado con las nuevas tecnologías BIM. El $90 \%$ cree que esta herramienta es más eficiente que el CAD, y que le permite reducir el tiempo de ejecución del proyecto, ya que se evitan los errores producidos en el sistema anterior. En las encuestas, se valora especialmente el hecho de aglutinar varias asignaturas, en torno a un modelado tridimensional único; ya que, el $99 \%$ de los encuestados cree recibir una docencia con mayor carácter integral y se pide que esta dinámica se haga extensible a otras asignaturas del grado.

\section{Conclusiones}

Por un lado, el uso de las Tecnologías BIM, da consistencia a los proyectos. Cuando los alumnos realizan un cambio en el proyecto a través del modelo tridimensional paramétrico, éste aparece reflejado en planta, alzado, sección... por tanto siempre habrá una consistencia entre todos los documentos que forman el proyecto, porque solo existe un único objeto base. Hemos constatado por tanto, una mayor velocidad en la ejecución de los proyectos (ahorro de un $15 \%$ en los tiempos de ejecución). Se aprecia un resultado de mayor precisión, y con unas calidades gráficas inusitadas.

Por otro, el hecho de que compartan dicho modelado tridimensional entre asignaturas, permite evitar solapes de temario entre las mismas. No se da una duplicidad de requerimiento de adquisición de competencias y, por tanto, el alumnado dispone de recursos temporales más amplios para incidir en su aprendizaje, alcanzando calidades de resolución más elevadas.

Este modelo tridimensional único evita errores en los planos, ya que se corrigen durante el diseño del modelo (en cualquiera de sus vistas), y esto hace que, se actualicen de forma automática todos planos del proyecto. Ello repercute positivamente en una disminución tanto de problemas, como de gastos en la ejecución. Este proceso conlleva una reducción de coste en el proyecto, pero sobre todo una mejora del rendimiento, reduciendo el tiempo utilizado en la realización del proyecto, ya que los intervinientes, no participan de forma secuencial, uno tras otro, sino que lo hacen de una forma concurrente, participando de forma simultánea, pudiendo realizar un trabajo colaborativo, gracias a los sistemas de participación en grupo (Hernández 2011).

Las instituciones de educación en general, tienen las opciones de adaptación de sus planes de estudio existentes para incluir BIM o, enseñar BIM como curso separado o ambos (Sacks y Barak, 2009).

Uno de los grandes hándicaps de esta metodología es la necesidad de formación del resto del profesorado del grado, de forma que todas las asignaturas puedan estar integradas en el desarrollo del proyecto sobre un mismo modelo tridimensional. Es habitual que al profesorado que decide realizar la formación inicial en la herramienta, por un lado le cueste encontrar la seguridad necesaria para enfrentarse al reto de la enseñanza aplicada en su asignatura; por otro, se resista a enfrentarse a la tesitura de tener que replantearse toda su asignatura para poder integrarse en una nueva forma de enseñanza basada en el reto de que el alumnado sea 
el centro de un aprendizaje activo, donde la cooperación y la colaboración son pilares sobre los que se asienta este nuevo aprendizaje.

También es cierto, que los grados derivados del proceso de Bolonia, en muchos casos, acaban de pasar la acreditación quinquenal, pero no tienen recogidas en sus memorias de verificación el empleo de esta nueva tecnología BIM. Esto hace que el profesorado que está dispuesto a realizar el cambio, se halle en una situación de incertidumbre ante el cambio, pero además, hace que el profesorado que no está por la labor del cambio, halle una excusa perfecta para mantener su antigua situación, de forma que el proceso de enseñanza colaborativa se quede parcialmente cojo.

Es deber de las universidades para adaptarse adecuadamente a las nuevas exigencias de la sociedad, que actualice sus planes de estudio a la vez que a sus egresados en profesionales, como los especialistas necesarios (Barison y Santos 2010).

\section{Referencias}

BARISON, M. B., y SANTOS, E. T. (2010). "Review and analysis of current strategies for planning a BIM curriculum". En: CIB W78 2010 27th International Conference. São Paulo: University of São Paulo. 1-10.

BOEYKENS, S. et al. (2013). "Experiencing BIM Collaboration in Education" Proceedings of the 31st eCAADe Conference. Delft: Delft University of Technology, vol. 2, p. 505-513.

EASTMAN, C. et al. (2011). BIM handbook: A guide to building information modeling for owners, managers, designers, engineers and contractors. New Yersey: John Wiley \& Sons.

GU, N. \& DE VRIES, B. (2012). "Two Approaches to Implementing BIM in Architectural Curricula". Proceedings of the 30th eCAADe Conference. Czech Republic: Technical University in Prague. vol. 1, p. 39- 48.

HERNANDEZ, L.A. (2011). "Hacia el proyecto digital" en EGA: revista de expresión gráfica arquitectónica, vol. 18 , p. $270-279$.

PENTTILÄ, H. \& WECK, TU. (2006) "The effects of information and communication technology (ICT) on architectural profession." ECPPM-e-Business and e-work in Architecture, Engineering and Construction. Helsinki: Helsinki University of Technology.

PRENSKY, M. (2001) "Digital natives, digital immigrants" en On the Horizon, vol. 9, no 5, p. 1-6.

SACKS, R.; BARAK, R. (2009) "Teaching building information modeling as an integral part of freshman year civil engineering education" en Journal of professional issues in engineering education and practice, vol. 136 , no 1 , p. 30-38. 